\title{
Design of signal and system virtual teaching course platform based on LabVIEW
}

\author{
Haima Yang ${ }^{1, *}$, Xianghong Zhang ${ }^{1}$, Gang Fang ${ }^{1}$, Jun $\mathrm{Li}^{2}$, Dawei Zhang ${ }^{1}$, Jin Liu ${ }^{3}$, \\ and Jie Ying ${ }^{1}$ \\ ${ }^{1}$ School of Optoelectronics, University of Shanghai for Science and Technology, Shanghai, China \\ ${ }^{2}$ Sino-German College of Shanghai for Science and Technology University, Shanghai, China \\ ${ }^{3}$ School of Electronic and Electrical Engineering, Shanghai University of Engineering Science, \\ Shanghai, China
}

\begin{abstract}
Signals and Systems" is the core professional course of electronic information majors. Aiming at its characteristics of strong theory, abstract content, and high memory requirements, a virtual teaching platform based on LabVIEW is designed to strengthen students' understanding of this course It is of great significance to reduce the difficulty of students' learning, improve students' learning efficiency, and stimulate students' interest in learning. At the same time, with the synchronous teaching of relevant course experiments, it can greatly reduce the difficulty of teachers' teaching and improve the quality of teaching.
\end{abstract}

\section{Introduction}

"Signals and Systems" is a core course of related majors such as electronic information and communication engineering in colleges and universities. It has the characteristics of wide knowledge, strong theory and abstract concepts. At the same time, the content of the course is complex, there are many formulas and theorems that need to be memorized and understood, and the amount of exercises is huge. It has brought certain difficulties to teachers' experimental teaching. It is also challenging for students to learn this course well. In the 21 st century with the rapid development of various science and technology, how to use new scientific and technological achievements in experimental teaching is While reducing the teaching difficulty of teachers, improving the learning efficiency and adaptability of students is a problem that requires us to constantly think and explore.

\section{Significance of using LabVIEW-based virtual teaching platform in signal and system courses}

In the traditional teaching of signal and system courses, teachers often use the teaching method of blackboard calculus in the classroom, resulting in a boring classroom atmosphere, and a large number of knowledge points cannot be vividly expressed through this teaching

\footnotetext{
* Corresponding author: snowyhm@sina.com.cn
} 
method, and students often apply a lot of applications Formula exercises training to consolidate and memorize the knowledge learned in the classroom, with less effort and less efficiency. At the same time, due to the large number of signal analysis results in the signal and system course, various complex waveform results will be generated, and small changes in conditions will cause huge changes in the results, Lack of simple and intuitive forms of expression on paper, resulting in a very weak connection between students' understanding of mathematical functions and actual signal waveforms, lack of direct perceptual understanding of experimental results, and poor learning results, which greatly dampens students' interest in learning, So that students are tired of coping with the course examinations and it is difficult to really learn the knowledge in this course and understand the significance of this course in professional learning.

The signal and system course is a difficult course in the learning process for students. For teachers, it is also the most important and difficult point in the teaching process. How to reduce the difficulty of teacher teaching, improve students' understanding, stimulate students' interest in learning, and deepen the interaction between teachers and students and the overall improvement of teaching quality are major issues that need to be resolved in the development of this course.

At this stage, major colleges and universities generally adopt a combination of theoretical teaching and course experiments to promote and improve students' understanding of course knowledge. The oscilloscope in the laboratory is used to show the occurrence of some signals and the process of waveform transformation. To a certain extent, it is helpful for students to understand the relevant signal transformation process, but it is difficult to comprehensively and systematically display the transformation process of various signals, and it is powerless for the transformation process of multiple signal superimposition, which has great limitations. At the same time, laboratory related equipment Due to the limited number and intense use, students cannot verify the learning results anytime and anywhere. At the same time, the related experimental equipment lacks maintenance, and the accuracy of the experimental results needs to be considered. Using special software to learn this course on a personal computer has great advantages.

In order to achieve the ideal teaching effect, we need to use teaching tools that are more suitable for this course. The teaching software written by LabVIEW can quickly, accurately and visually display the transformation process of various signals, and cooperate with carefully designed course experiments to reduce the number of teachers. While teaching tasks, it saves students' learning time and reduces the difficulty in understanding various formulas and theorems. The learning process is lively and interesting, which can greatly improve the quality of teaching in the process of driving students' interest in learning and strengthening teacher-student interaction.

\section{Use of LabVIEW-based virtual teaching platform}

The virtual teaching platform is developed based on LabVIEW software, which has powerful functions for signal processing. With the continuous development of modern computer information technology, virtual instruments have been applied to varying degrees in various fields. It mainly refers to the user-designed function on the computer hardware platform, and the test function is realized through the test software, which has certain convenience and flexibility. As one of the most successful virtual instrument software at present, LabVIEW is a graphical programming language, which uses 32-bit compiled G language. The LabVIEW interface is conducive to the realization of virtual instrument development, and it has multiple functions such as data acquisition, analysis, and signal processing., Has been widely used in many fields. 


\subsection{Main interface design}

As the main interface of the system, it must not only be beautiful, but also simple and clear. Entering the main interface first displays the contents of the three modules, and has the function of "clicking the module button to pop up a sub-interface window".

The main interface is to $\log$ in to the system, enter the correct account and password, and then select the teacher or student identity to log in. If the login is successful, it will jump to the selection interface. Otherwise, it will prompt login failure, and display the reason in the login result. The main interface of login system is shown in Figure 1.

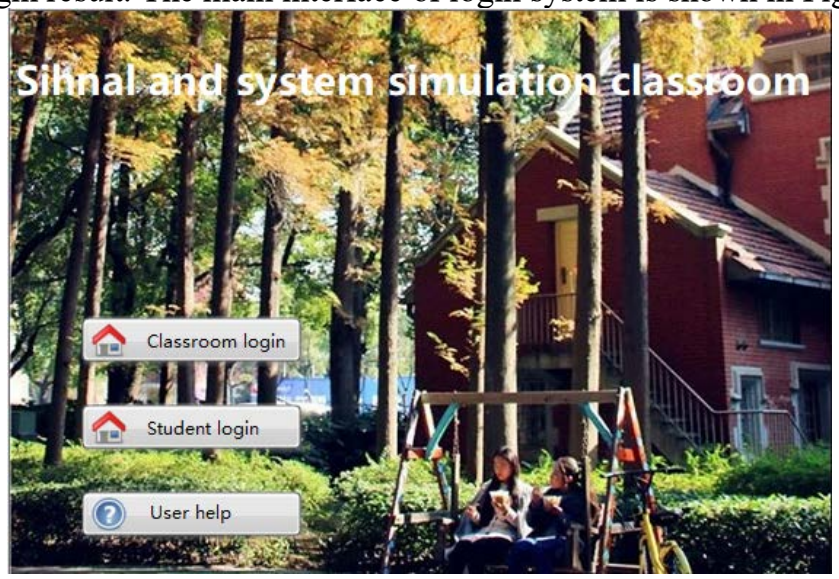

Fig. 1. System main interface.

According to the teaching requirements of the "Signals and Systems" course, the design and analysis of each content in the four teaching modules of time domain, transform domain, system analysis, and discrete system in continuous systems have been completed with LabVIEW. The front panel of the experiment selection interface is shown in Figure 2.

In this interface, you can choose to enter one of the seventeen teaching modules for demonstration, or choose to exit the system directly. Determine the branch of the event structure through the selection on the front panel, enter a different demo module or stop the demo. After the teaching demonstration, students can log in to the system and independently select the corresponding content for signal simulation experiments.

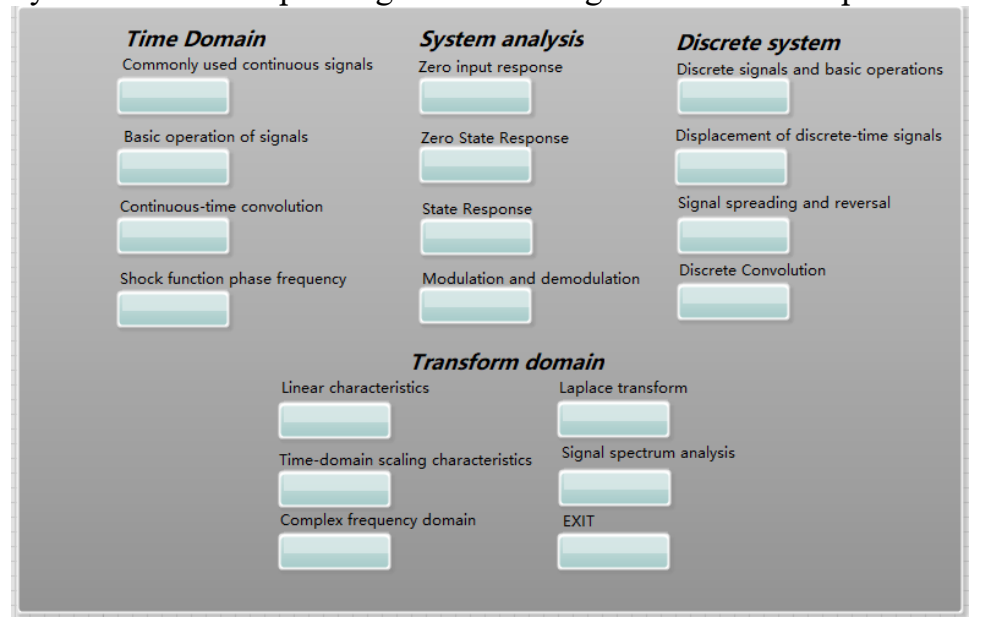

Fig. 2. Login system panel. 


\subsection{Part of the design module display}

In the analysis of continuous-time signals, most of the common signals can be represented by basic signals or their variations. Basic signals include DC signals, sine signals, exponential signals, step signals, etc. The simulation interface of commonly used continuous time signals is shown in the LabVIEW virtual front panel shown in Figure 3. This interface includes two major modules: input and display. The input module can be Select the type of signal (including exponential decay sine, sampling, real exponential, triangle, sine, square wave, sawtooth and other signals) and set the frequency, amplitude, phase, etc. of different signals.

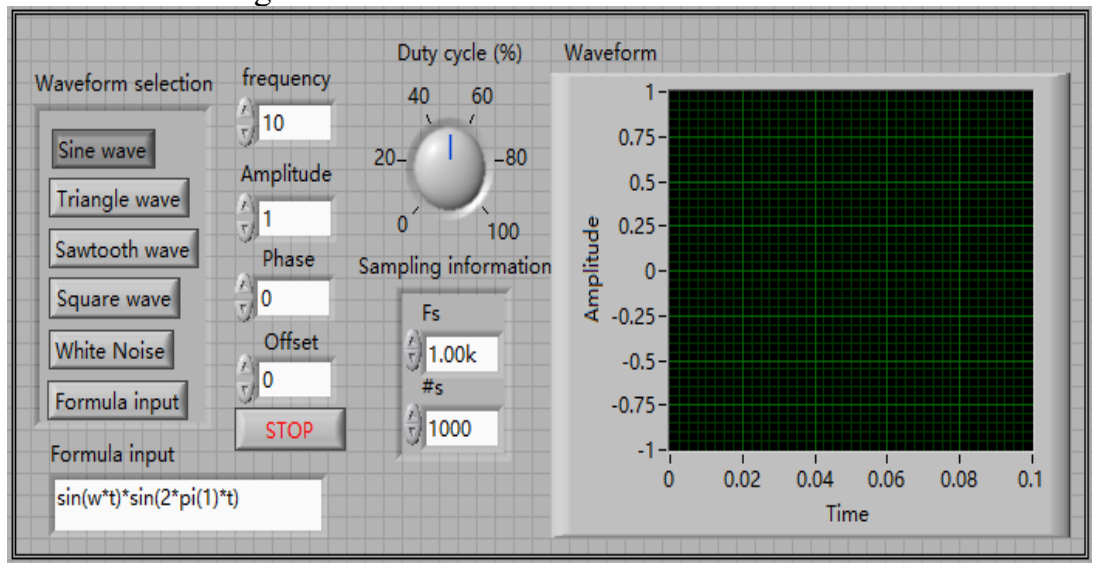

Fig. 3. Signal generator.

For simple filtering and noise processing, it is also common in signal processing. The interface is shown in Figure 4.

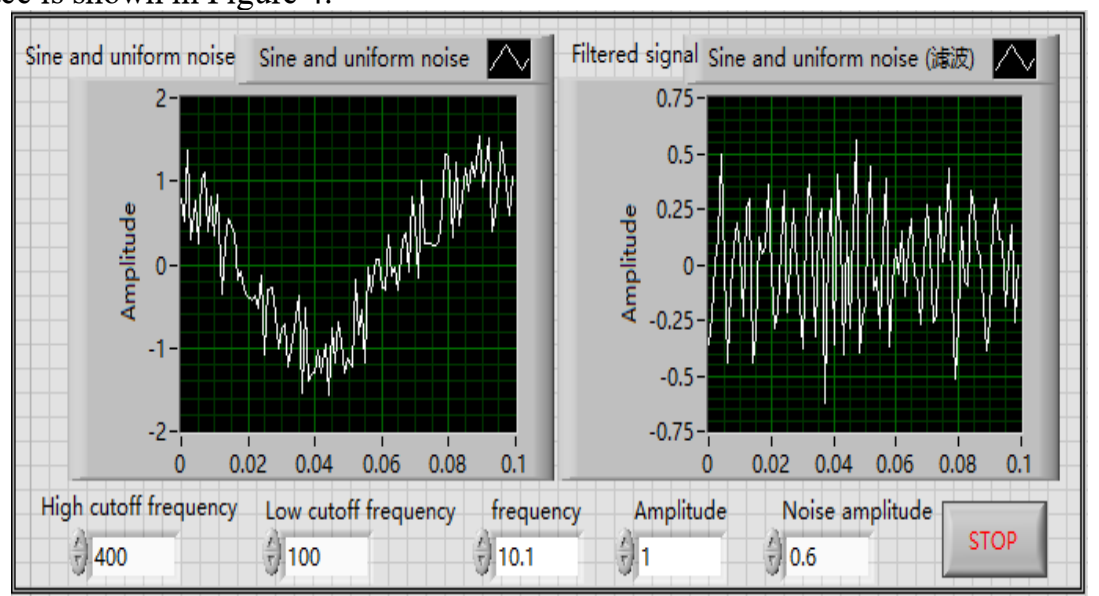

Fig. 4. Filter.

The basic operation interface of the signal is shown in Figure 5, which is also divided into input and display modules. The input module adds a waveform selection and operation control on the basis of commonly used continuous time signals to add, subtract, multiply, and multiply different waveforms. Operation of division and formula.

Signal modulation is to use the baseband signal to control the change of one or several parameters of the carrier signal, and load the information on it to form a modulated signal transmission, while demodulation is the inverse process of modulation, from the modulated 
signal through a specific method The original baseband signal will be restored when the parameter changes. For signal detection, it is also commonly used. We use LabVIEW for simulation. The program interface is shown in Figure 6 below.

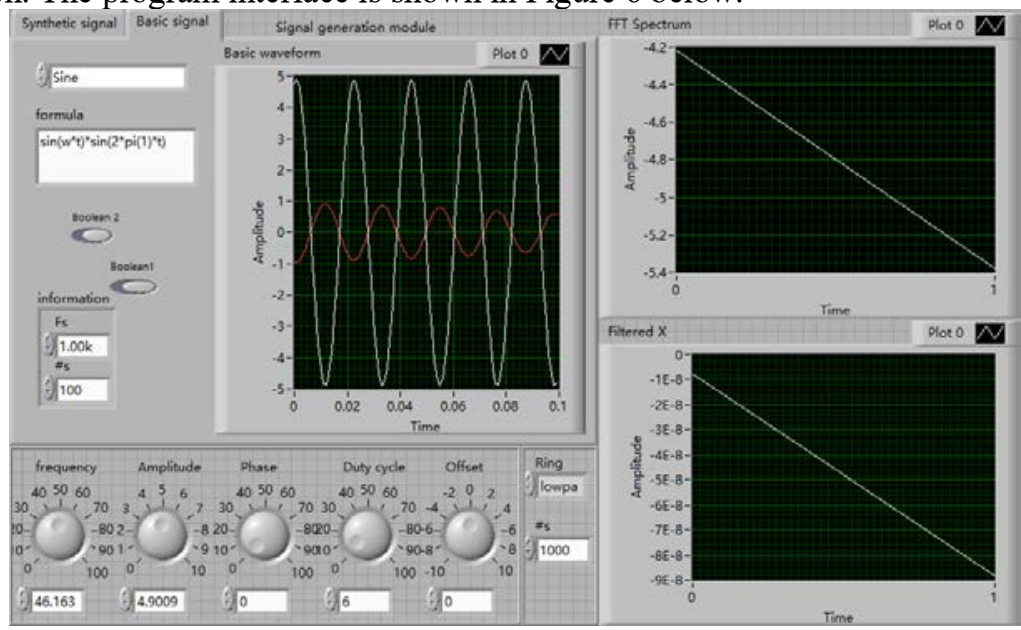

Fig. 5. Signal basic operation.

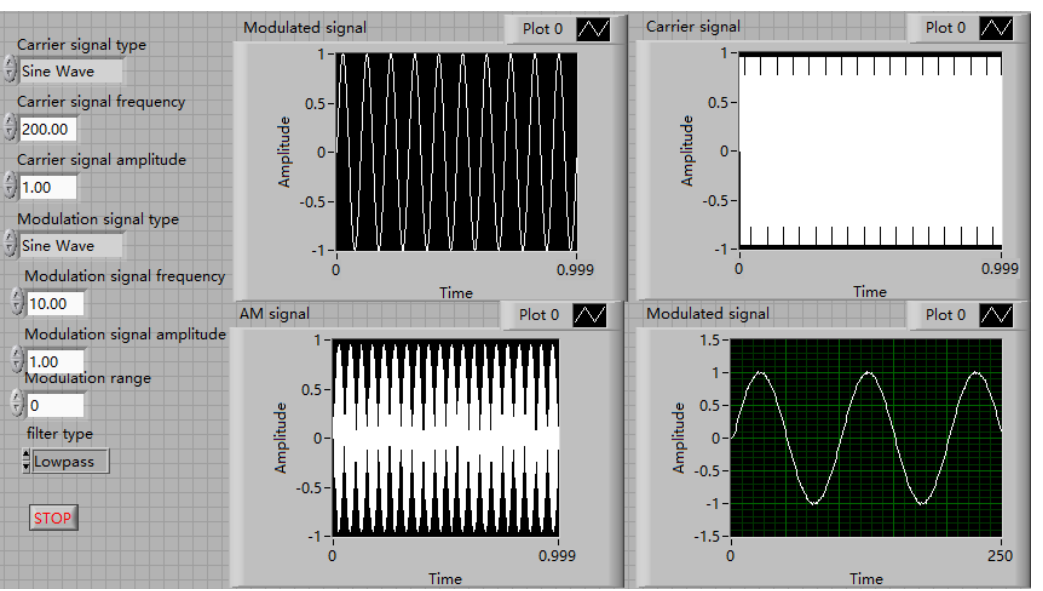

Fig. 6. Modulation and demodulation.

\section{Conclusion}

With LabVIEW as the main development environment, a software teaching platform for the 'Signals and Systems' course has been built, which can realize the intuitive demonstration and experimental teaching of important signals and key content in the course. It is intuitive and visual, which can effectively improve the teaching effect and improve students the initiative and the teacher's teaching efficiency. It is of great significance to improve the teaching effect of this course. It can provide useful references for other related courses.

Fund support: University of Shanghai for Science and Technology Teacher Development Project (CFTD203016); University of Shanghai for Science and Technology Virtual Simulation Experiment Teaching Cultivation Project (2020GD06) 


\section{References}

1. Wei Tianxing. Some thoughts on the cultivation of college students' innovative ability [J]. China Forestry Education, 2010 (1).

2. Zhong Yan. On the cultivation of college students' scientific and technological innovation ability [J]. Science and Technology Information, 2011 (1).

3. Liu Shuang. Talking about the cultivation of college students' scientific and technological innovation ability[J]. China Science and Technology, 2013 (22).

4. Zhou Jun, Chen Qi. On the cultivation of college students' scientific and technological innovation ability [J]. Journal of Guangxi Youth Leaders College.

5. Zhang Panpan. Research on the Path of Cultivating Innovative Talents [J]. Youth Years, 2011 (24).

6. Zhang Han, Zhang Jingmei. The interactive relationship between the cultivation of scientific and technological innovation ability of college students and the reform of education and teaching [J]. Science and Technology Vision, 2013 (27).

7. Wang Junli, Nie Guoxing. The status quo and countermeasures of higher education biotechnology specialty [J]. Journal of Henan Academy of Science and Technology, 2015 (05).

8. Huang Jing. The characteristics of talent training under the background of "Internet +" [J]. Science and Technology of Chinese Universities, 2017 (01). 